\title{
A comparison of facial emotion processing in neurological and psychiatric conditions
}

\author{
Benoit Bediou 1,2,3 *, Jérôme Brunelin 1,4 ,Thierry d'Amato ${ }^{1}$, Shirley Fecteau ${ }^{4,5}$, Mohamed Saoud ${ }^{1}$, \\ Marie-Anne Hénaff ${ }^{6}$ and Pierre Krolak-Salmon ${ }^{6,7}$ \\ 1 Université de Lyon, Lyon, France \\ ${ }^{2}$ Centre Hospitalier "Le Vinatier ", Bron, France \\ ${ }^{3}$ Swiss Center for Affective Sciences, University of Geneva, Geneva, Switzerland \\ ${ }^{4}$ Institut Universitaire en Santé Mentale de Québec, Laval University, QC, Canada \\ ${ }^{5}$ Department of Neurology, Berenson-Allen Center for Noninvasive Brain Stimulation, Beth Israel Deaconess Medical Center, Harvard Medical School, Boston, MA, \\ USA \\ ${ }^{6}$ INSERM, U1028, CNRS, UMR5292, Brain Dynamics and Cognition Team, Lyon Neuroscience Research Center, Lyon, France \\ 7 Memory Center of Lyon, Hospices Civils de Lyon, Hôpital des Charpennes, Villeurbanne, France
}

Edited by:

Marina A. Pavlova, Eberhard Karls University of Tübingen, Germany

Reviewed by:

David Terburg, Universiteit Utrecht, Netherlands

Linda Isaac, Radboud University, Netherlands

${ }^{*}$ Correspondence:

Benoit Bediou, Brain and Learning Laboratory, Faculty of Psychology, Université de Genève, Boulevard Pont d'Arve, 40, CH-1205 Geneva,

Switzerland.

e-mail: benoit.bediou@unige.ch
Patients suffering from various neurological and psychiatric disorders show different levels of facial emotion recognition (FER) impairment, sometimes from the early phases of the disease. Investigating the relative severity of deficits in FER across different clinical and high-risk populations has potential implications for the diagnosis and treatment of these diseases, and could also allow us to understand the neurobiological mechanisms of emotion perception itself. To investigate the role of the dopaminergic system and of the frontotemporal network in FER, we reanalyzed and compared data from four of our previous studies investigating FER performance in patients with frontotemporal dysfunctions and/or dopaminergic system abnormalities at different stages. The performance of patients was compared to the performance obtained by a specific group of matched healthy controls using Cohen's $d$ effect size. We thus compared emotion and gender recognition in patients with frontotemporal dementia (FTD), amnestic mild cognitive impairment (aMCl), Alzheimer's disease (AD) at the mild dementia stage, major depressive disorder, Parkinson's disease treated by I-DOPA (PD-ON) or not (PD-OFF), remitted schizophrenia (SCZ-rem), first-episode schizophrenia treated by antipsychotic medication (SCZ-ON), and drug-naïve first-episode schizophrenia (SCZ-OFF), as well as in unaffected siblings of patients with schizophrenia (SIB). The analyses revealed a pattern of differential impairment of emotion (but not gender) recognition across pathological conditions. On the one hand, dopaminergic medication seems not to modify the moderate deficits observed in SCZ and PD groups (ON vs. OFF), suggesting that the deficit is independent from the dopaminergic system. On the other hand, the observed increase in effect size of the deficit among the aMCl, $A D$, and FTD groups (and also among the SIB and SCZ-rem groups) suggests that the deficit is dependent on neurodegeneration of the frontotemporal neural networks. Our transnosographic approach combining clinical and high-risk populations with the impact of medication provides new information on the trajectory of impaired emotion perception in neuropsychiatric conditions, and on the role of the dopaminergic system and the frontotemporal network in emotion perception.

Keywords: facial expression recognition, psychiatry, neurology, social cognition, transnosographic approach

\section{INTRODUCTION}

Emotions play a central role in decision-making and adaptation in humans. Dysfunction of the emotional regulation system is involved in behavioral and pathological issues, including aggressiveness, addiction, and risk-taking behavior, as well as anxiety and depression (Davidson et al., 2000; Dalgleish, 2004). Among social cognitive functions, emotion perception has attracted increasing interest over the last two decades. Emotional stimuli affect basic and cognitive operations such as perception, attention and memory, and altered emotion perception is associated with a variety of neurological and psychiatric conditions (Phillips and David, 2003; Kohler et al., 2004).

\section{THE NEURAL BASES OF THE RECOGNITION OF FACIAL EXPRESSION Frontotemporal networks}

Neuroimaging studies have provided crucial information regarding the neural processes of emotion recognition by showing that the frontotemporal brain networks are involved in the processing of faces and facial expressions (Haxby et al., 2000; Kanwisher and Moskovitch, 2000; Eimer and Holmes, 2007). Accordingly, 
a decrease in the ability to recognize facial expressions has been reported in patients with frontotemporal dementia (FTD, Keane et al., 2002; Rosen et al., 2004) and has been linked to the transformation from the amnesic mild cognitive impairment (aMCI) stage toward the dementia stage of Alzheimer's disease (AD, Spoletini et al., 2008; Bediou et al., 2009a). This transformation is associated with wider frontotemporal lesions (Braak and Braak, 1991). The deficit has also been reported in Williams syndrome, a rare genetic disorder with disrupted brain networks involving the frontal and temporal cortex (Isaac and Lincoln, 2011). Impaired emotion recognition in patients with schizophrenia and their siblings is associated with disturbed brain activity in frontotemporal networks (Streit et al., 2001; Ioannides et al., 2004; Bediou et al., 2007b; Seiferth et al., 2008). Finally, the deficit is also present in patients with major depressive disorder (MDD; Feinberg et al., 1986; Gur et al., 1992; Rubinow and Post, 1992; Persad and Polivy, 1993; however, see Bediou et al., 2007a for a null result; for a recent review of emotion recognition in MDD, see Bediou et al., 2009b), where it is associated with abnormal activity in frontotemporal regions (Sheline et al., 2001; Fu et al., 2004, 2007; Lawrence et al., 2004; Canli et al., 2005; Chan et al., 2009).

\section{Dopamine}

Emotion recognition has also been linked to the dopaminergic system (Delaveau et al., 2005; Salgado-Pineda et al., 2005). A decrease in the ability to recognize facial expressions has been associated with diseases that are closely linked to dopaminergic dysfunctions such as Parkinson's disease (PD, Dujardin et al., 2004; LachenalChevallet et al., 2006; Lawrence et al., 2007; Clark et al., 2008) and schizophrenia (SCZ, Zuroff and Colussy, 1986; Heimberg et al., 1992; Lewis and Garver, 1995; Wolwer et al., 1996; Streit et al., 1997; Bediou et al., 2005a, 2007a). The deficit has also been observed in subjects with a heightened risk for schizophrenia (Poreh et al., 1994; Toomey and Schuldberg, 1995; Mikhailova et al., 1996; Van 'T Wout et al., 2004; Bediou et al., 2007a) as well as in other clinical and non-clinical samples of high-risk individuals (e.g., those with social anxiety, schizotypal personality disorder, see Heuer et al., 2010; Germine and Hooker, 2011), who exhibit abnormal dopamine transmission (Tost et al., 2010).

\section{Clinical studies}

Although impaired facial expression processing had already been extensively reported in FTD (Lavenu et al., 1999; Keane et al., 2002; Rosen et al., 2004) and in schizophrenia (Zuroff and Colussy, 1986; Heimberg et al., 1992; Wolwer et al., 1996; for a review, see Mandal et al., 1998), the results are far less consistent in subjects who are at risk for schizophrenia (Bolte and Poustka, 2003; for a review, see Phillips and Seidman, 2008), in AD (Cadieux and Greve, 1997; Roudier et al., 1998; Bucks and Radford, 2004), in MDD (for a review, see Bediou et al., 2009b), in mild cognitive impairment (Spoletini et al., 2008), in prodromal ALZ (Kohler et al., 2005), and in PD (Assogna et al., 2008).

To the best of our knowledge, the relative extent of emotion recognition deficits across different clinical groups has not yet been investigated. This dearth is partly due to the heterogeneity of the methods used to investigate the same function in previous research. Most of the studies on emotion perception have emerged from different groups using very different stimuli and paradigms in different normal and/or clinical populations. Moreover, when used, control tasks have been another source of methodological variation across studies. Consequently, it has remained difficult to address the relative extent of the impairments in emotion recognition across different pathologies.

\section{THE PRESENT STUDY}

In a series of studies using the same stimuli and paradigm (Bediou et al., 2005a,b, 2007a, 2009a; Lachenal-Chevallet et al., 2006), we have investigated facial expression recognition in various neurological and psychiatric conditions including SCZ, MDD, PD, $\mathrm{AD}$, and FTD. In addition, facial expression recognition has been studied in non-clinical populations such as unaffected siblings of schizophrenics (SIB) and subjects with aMCI. In each study, the performance of the studied group was compared to that of a group of healthy subjects of comparable sex ratio, age, and educational level. In all studies, gender recognition was used as a control task in order to compare the processing of socially significant changeable facial features to that of a more physically invariant facial feature. These studies have shown that a deficit in emotion but not in gender recognition was observed in several conditions. Using the same protocol in multiple neurological and psychiatric groups provides a unique opportunity to compare these populations with regard to their ability to recognize facial expression. We used a statistical method based on the comparison of effect sizes using Cohen's $d$ coefficient to compare emotion recognition performances among these neuropsychiatric diseases that lead to early social behavior disturbances with those observed in their matched control groups. The strengths of our method reside in the use of morphed faces, which increases the sensitivity of the measurements for both the emotion and the gender recognition tasks.

Extensive and sometimes inconsistent literature has tried to link emotion-specific deficits in patients with anomalies in circumscribed neural systems. Among the most famous examples are the relationships between (a) fear and the amygdala (e.g., Adolphs et al., 1994; Krolak-Salmon et al., 2004) and the (b) putamen/insula and disgust (e.g., Calder et al., 2000; Krolak-Salmon et al., 2003). The amygdala and the insula/basal ganglia are affected in various neurological and psychiatric conditions, including schizophrenia and obsessive-compulsive disorder, where these brain anomalies may contribute to impaired face-emotion processing (for a more thorough discussion, see Sprengelmeyer et al., 1997; Aleman and Kahn, 2005; Shayegan and Stahl, 2005; Van Rijn et al., 2005). Importantly, however, differential or emotion-specific impairments may not necessarily reflect specific neural anomalies and may arise from differences in low-level properties of the stimuli (e.g., Calder et al., 2001; Deruelle and Fagot, 2005) or from attentional or cognitive deficits (Adolphs et al., 2005).

Importantly, the goal of the present study was NOT to describe emotion-specific recognition deficits in particular populations in detail. Instead, our aim here was to compare, for the first time, the extent of the overall emotion recognition deficits across the various clinical and high-risk populations that were tested in our previous studies. To increase the power of our statistical analysis, we focused on overall emotion and gender recognition performance (i.e., 
collapsed across all emotions/genders and all morphing levels). We did not analyze each emotion or morphing level separately as this may have resulted in low statistical power. Moreover, detailed information about the emotions affected in each population can be found in the relevant literature. In the present study, we focus on the comparison of overall emotion recognition abilities across clinical and high-risk populations.

Such a transnosographic investigation of emotion perception across different clinical and high-risk populations is an important challenge that has potential implications not only for the prevention, diagnosis, and treatment of these diseases but also for our understanding of the neurobiological mechanisms of emotion perception itself. On a neurobiological level, understanding the relationship between impaired emotion perception and the severity of a disorder may help elucidate the neural networks or neurotransmitter activity involved in either the disorder or the mechanisms of emotion perception per se. First, examining emotion recognition in aMCI and mild $\mathrm{AD}$ may clarify the impact of neurodegenerative processes in particular neural structures especially the amygdala, which is critical for emotion perception (Adolphs et al., 1994). Second, studying emotion perception abilities in SCZ and PD and assessing the impact of medication may elucidate the links between emotion recognition and dopaminergic neurotransmission (Salgado-Pineda et al., 2005) and the role of the mesocorticolimbic and nigrostriatal circuits that are differentially affected by these pathologies (Deutch, 1993). Third, on a clinical level, discovering a trajectory of impaired emotion perception may help in early differential diagnosis and assist in treatment planning, potentially including early intervention and prevention. For example, emotion recognition abilities may help to differentiate between atypical depression, schizophrenia, and subjects at risk and to detect the conversion from aMCI or prodromal AD to dementia states, which are critical to planning an appropriate intervention.

\section{MATERIALS AND METHODS SUBJECTS}

The participants involved in the present study included patients with schizophrenia in remission (SCZ-rem, $N=29$ ); patients experiencing their first-episode, in either the drug-naïve state
(SCZ-ON, $N=40$ ) or after they had taken medication (SCZ-OFF, $N=40)$; unaffected siblings of schizophrenia patients $(N=30)$; patients with $\mathrm{MDD}(N=20)$; PD patients $\mathrm{ON}$ (PD-ON, $N=12$ ) or OFF (PD-OFF, $N=13$ ) L-DOPA treatment; patients with mild $\operatorname{AD}(N=10)$; patients with FTD $(N=10)$; and patients with aMCI $(N=10)$. Patients were recruited at the psychiatric department at Le Vinatier hospital and at the neurological department at Hospital Pierre Wertheimer in Lyon (France). First-episode SCZ patients and their unaffected siblings (SIB) were recruited at the Ibn Nafis Psychiatric Hospital in Marrakech (Morocco). Healthy controls were recruited from the general population. Diagnoses were based on respective consensual criteria at the time of assessment. SCZ and MDD patients were diagnosed according to the Diagnostic and Statistical Manual (DSM) of Mental Disorders IV criteria (American Psychiatric Association, 1994); idiopathic PD was diagnosed according to the criteria of the United Kingdom PD brain bank (Gibb and Lees, 1989). Other patients were diagnosed with the appropriate consensus criteria for an aMCI (Petersen et al., 1999), mild dementia related to AD (McKhann et al., 1984), and FTD (Neary et al., 1998). The age and gender repartition of the patients as well as of their respective control groups in the original studies are presented in Table 1. Importantly, in each study, there was no difference in age or gender repartition between the clinical and the control groups. In addition, participants in each study completed a series of behavioral measures and questionnaires as well as a battery of cognitive tests. However, we did not collect the same information in all groups and only focused on measures that were relevant to the studied population. When available, information about disease onset and duration and participants' descriptions including behavioral and clinical evaluations and questionnaires are provided in these specific previous studies (see Table 1). We did not exclude any participant from any study. There was no clear outlier; thus, all participants from all studies were included in this reanalysis.

\section{TASKS}

Two different tasks were performed: a facial expression recognition task and a gender recognition task. The order of the tasks was counterbalanced across participants in each group. Both tasks were performed using morphed faces varying in terms of the intensity

Table 1 | The ages and gender of participants in each study.

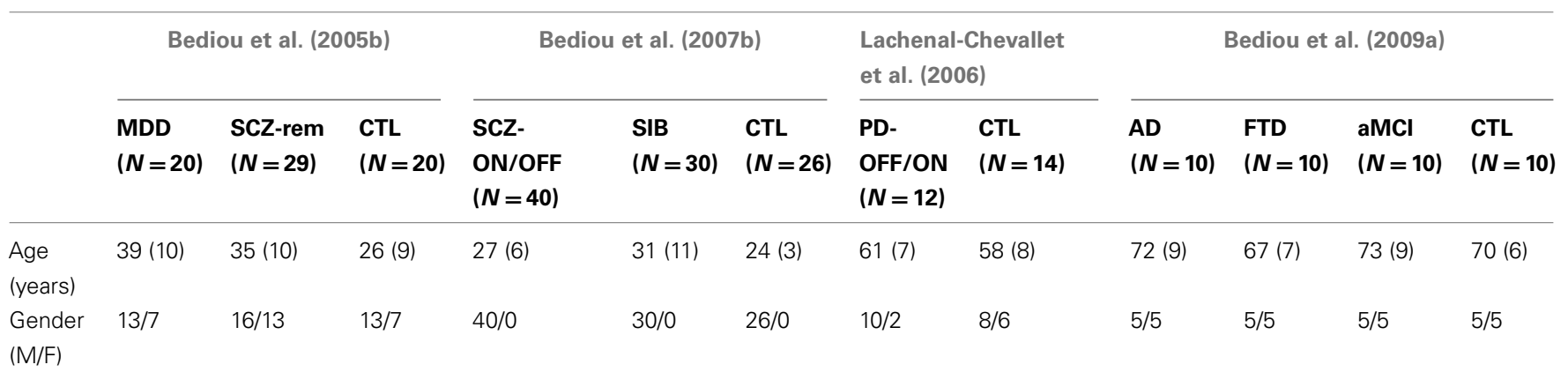

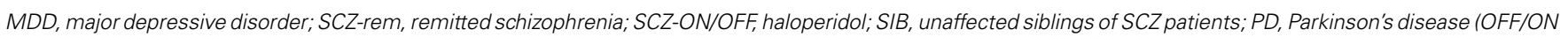
refer to I-DOPA treatment); $A D$, Alzheimer's disease at mild dementia stage; FTD, frontotemporal dementia; aMCI, (clinical) amnestic mild cognitive impairment (i.e., prodromal $A D) ; C T L$, controls. $M$, male; $F$, female. 
of the task-relevant face feature (Figure 1). In each task, subjects had to determine the expression or gender of each face using a forced-choice paradigm. For the facial expression recognition task, photographs of two female and two male faces depicting basic emotions (happiness, fear, anger, and disgust) morphed with a neutral face in $10 \%$ steps were randomly presented (the duration varied slightly across studies, from $400 \mathrm{~ms}$ to $1 \mathrm{~s}$ ). After each face presentation, participants were asked to decide which of five labels (happy, fearful, angry, disgusted, or neutral) best described the presented facial expression using a forced-choice paradigm. In the gender recognition task, photographs of the faces of eight females and eight males morphed with an average face in $10 \%$ steps were randomly presented. Each face had a neutral expression. After each face presentation, participants indicated the gender of the face using a forced-choice paradigm (man or woman).

The duration of stimulus presentation varied across studies (400 or $1000 \mathrm{~ms}$ ) and was always the same between the expression and gender recognition tasks. Although expression and gender morphs were performed using 10\% steps, studies in patients with SCZ and MDD revealed that using 20\% steps yielded similar results. Moreover, pilot testing revealed that increasing the stimulus duration from $400 \mathrm{~ms}$ to $1 \mathrm{~s}$ did not affect the judgments of these subjects but rather increased their confidence in these judgments, making the task less frustrating for them. We therefore used $20 \%$ steps and longer stimulus durations $(1000 \mathrm{~ms})$ in our studies comparing neurological patients to controls (i.e., AD, FTD, aMCI, and PD). These patients, unlike SCZ, MDD, and SIB patients, could not be transported to our experimental room and were tested in their own hospitalization rooms.

\section{STATISTICAL ANALYSIS}

The face-emotion and gender recognition performances in each group compared to the respective age- and sex-matched control group are shown in Table 1. Detailed information about participants and their performances have previously been published (Bediou et al., 2005b, 2007a, 2009a; Lachenal-Chevallet et al., 2006). In this study, we reanalyzed the data of these selected studies using a statistical approach borrowed from meta-analysis. However, given that we only focus on studies from our group, a calculation of publication bias based on the Fail Safe $N$ was

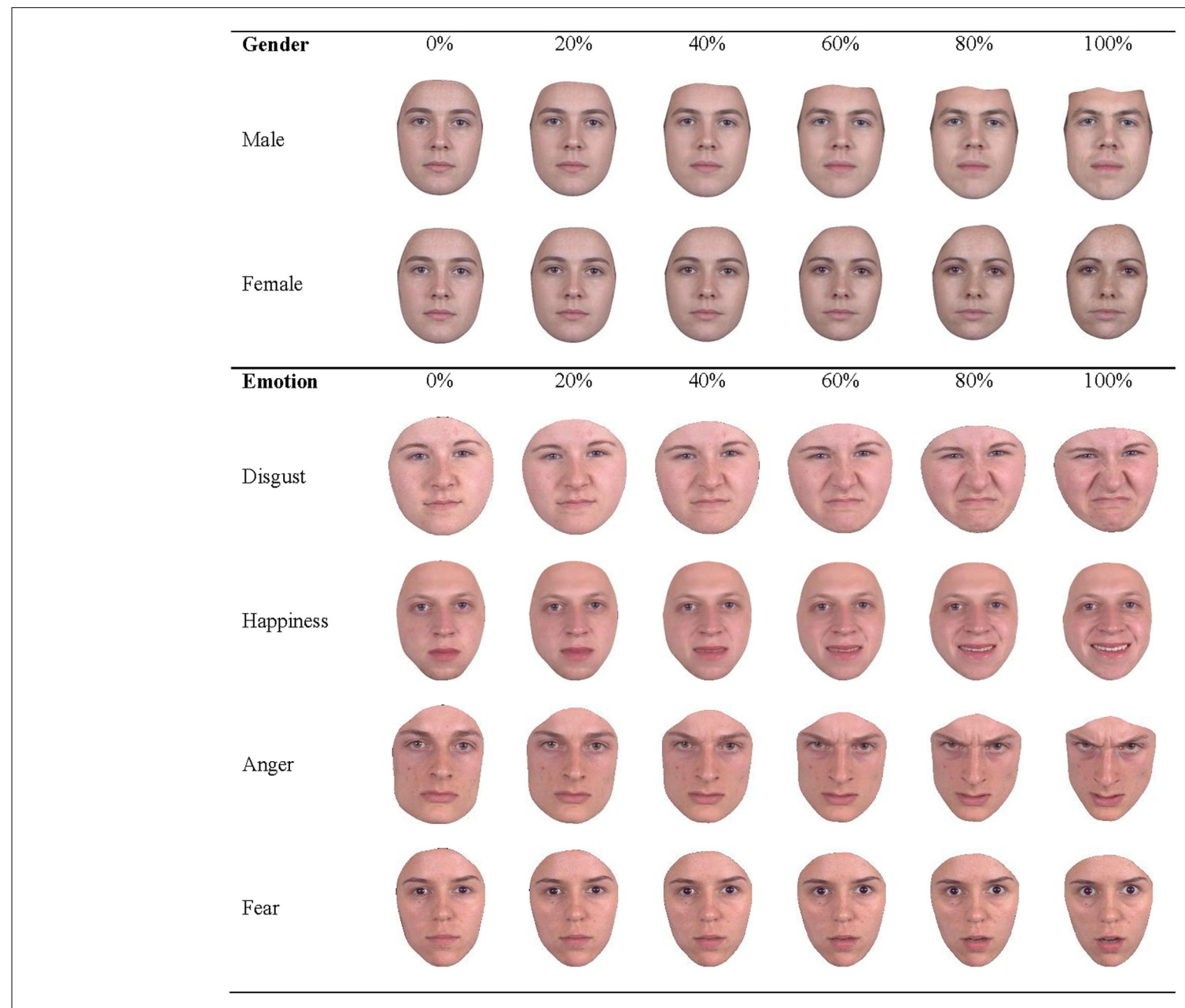

FIGURE 1 | Facial morphs used in the experiments. 
not appropriate. We thus invite cautious interpretation of our results.

Intra-group comparisons were assessed using Cohen's $d$ effect size measuring the percentage of variation in emotion and gender recognition performances between groups as compared to their own age- and sex-matched controls ${ }^{1}$. Cohen's $d$ is a standardized, scale-free measure of the effect size of the mean difference between two populations (Cohen, 1969). An effect size is exactly equivalent to a " $Z$-score" of a standard normal distribution. As suggested by Cohen (1969), an effect size of 0.2 could be considered to be small, 0.5 to be medium, and 0.8 to be large. An effect size of -0.5 indicates that the mean of the patient group is 0.5 SDs below the mean of the control group. The effect size estimate was corrected using a factor provided by Hedges and Olkin (1985). Both the mean and the confidence interval are important in the interpretation of Cohen's $d$; the mean corresponds to the magnitude, whereas the confidence interval reflects the significance of the difference. Importantly, the difference is statistically significant only if the confidence interval does not include zero. To more formally address the statistical significance of betweengroup differences, the calculation of Cohen's $d$ was followed by a corrected two-tailed Student's $t$-test for mean difference comparison between patients and the matched control group in each situation.

${ }^{1}$ To increase statistical power, emotions, and morphing levels were collapsed. Detailed statistical analyses including the morphing levels and emotions are available in corresponding papers, where emotion-specific deficits are discussed more thoroughly (see also Kohler et al., 2003).
Then, the inter-group differences were examined using a twotailed Student's $t$-test for comparison of the mean differences between groups of patients.

\section{RESULTS}

Gender recognition was preserved in all conditions as depicted in Figure 2, which presents the standardized and bias-corrected effect sizes as well as confidence intervals for emotion and gender recognition performance in each group. In contrast, emotion recognition was impaired compared to controls in drug-naïve first-episode SCZ patients, before treatment onset [SCZ-OFF, $d$ corrected $=-1.40(-1.95 ;-0.86) ; p$ corrected $<0.001]$ and after the clinical remission of symptoms [SCZ-ON, $d$ corrected $=-1.35$ $(-1.90 ;-0.80)$; $p$ corrected $<0.0001]$ using classical antipsychotic treatment, in clinically remitted SCZ patients receiving atypical antipsychotic treatments [SCZ-rem., $d$ corrected $=-0.81$ $(-1.41 ;-0.22) ; p$ corrected $<0.01]$ as well as in SIB [ $d$ corrected $=-0.68(-1.22 ;-0.14) ; p$ corrected $=0.01]$. PD patients were also impaired relative to controls during either the OFF phase [PD-OFF, $d$ corrected $=-1.54(-2.41 ;-0.66)$; $p$ corrected $<0.001]$ or the $\mathrm{ON}$ phase $[\mathrm{PD}-\mathrm{ON}, d$ corrected $=-1.56$ $(-2.44 ;-0.68) ; p$ corrected $=0.03]$ of L-DOPA treatment. Finally, patients with $\mathrm{AD}[d$ corrected $=-0.98(-1.91 ;-0.05)$; $p$ corrected $<0.001]$ and patients with FTD $[d$ corrected $=-2.54$ $(-3.71 ;-1.36) ; p$ corrected $<0.0001]$ also exhibited a deficit in emotion recognition performance compared to controls. In contrast, subjects with aMCI $[d$ corrected $=-0.33(-1.21 ; 0.55)$; $p$ corrected $=0.4, \mathrm{~ns}]$ as well as patients with MDD $[d$ corrected $=-0.42(-1.05 ; 0.20) ; p$ corrected $=0.2, \mathrm{~ns}]$ were not impaired relative to their respective control groups.

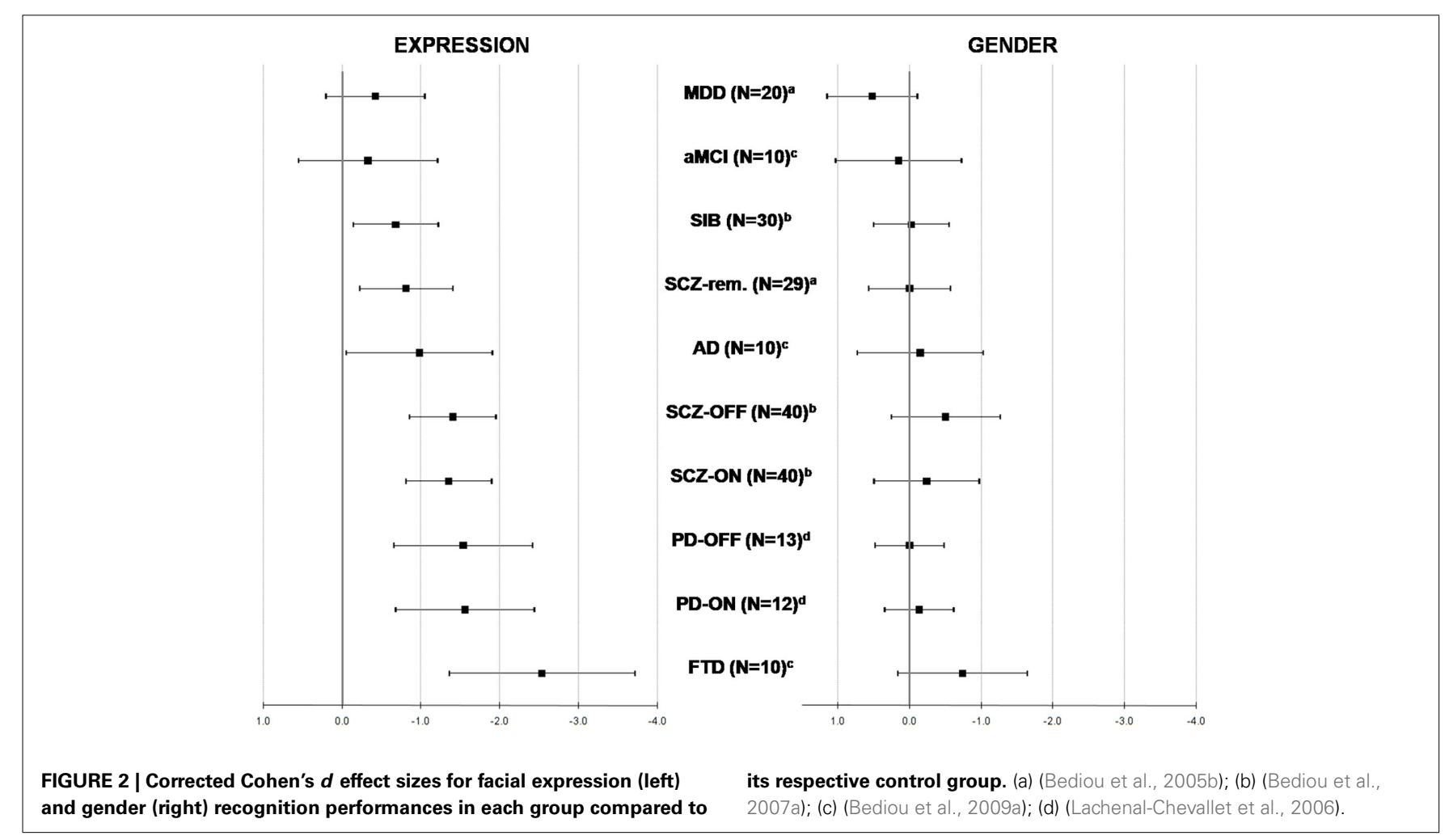


Concerning inter-group comparisons, we reported no difference in performance between the group composed of $\mathrm{AD}$, SIB, SCZ-rem, MDD, and aMCI patients. The performance of this group was significantly different from the performance of a group comprising SCZ-OFF, SCZ-ON, PD-ON, and PD-OFF $(p=0.002)$ patients. This last group differed significantly in terms of the performance of FTD $(p<0.0001)$.

\section{DISCUSSION}

This study aimed to compare emotion recognition deficits over different neurological and psychiatric conditions that involve early social behavioral disorders. Four groups of patients could be distinguished compared to their respective controls on the basis of emotion recognition abilities as measured by Cohen's $d$ value: (i) participants with MDD and aMCI did not present with any significant deficit $(d<0.5$; ns); (ii) SCZ-rem and SIB as well as $\mathrm{AD}$ patients presented with a mild impairment $(0.5<d<1)$; (iii) SCZ-OFF, SCZ-ON, PD-OFF and PD-ON patients presented with moderate impairment $(1<d<2)$; and (iv) FTD patients presented with a large impairment $(d>2.5)$. Importantly, gender recognition did not differ from controls in any of the groups, which suggests that the impairment is relatively selective to the processing of emotional or social cues. These results have implications for researchers who are interested in the neurobiological processes involved in emotion perception and recognition, and also for clinicians interested in the diagnosis and treatment of pathologies associated with impairment in this function.

As mentioned in the methods section, stimuli of longer durations were used in neurological patients to accommodate their comfort levels. Although pilot testing did not reveal any difference in performance and only increased confidence in their judgments, there are important differences between these two types of presentations. While a stimulus that is $400 \mathrm{~ms}$ in duration may delve into the automatic processing of faces, the longer 1000-ms duration may involve more elaborative and controlled processing of faces. This view is supported by EEG studies showing that early (e.g., P1, N170) and late components (e.g., P300, late positive potential) are sensitive to different information in the face, including differential sensitivity to emotion (e.g., Krolak-Salmon et al., 2001, 2003, 2004, 2006; for a review, see Pourtois and Vuilleumier, 2006; Eimer and Holmes, 2007; Vuilleumier and Pourtois, 2007; Pourtois and Vuilleumier, 2010).

\section{IMPLICATIONS FOR THE NEUROBIOLOGICAL MECHANISMS OF EMOTION PERCEPTION}

Recent work has linked emotion recognition deficits to the disturbance of a frontotemporal brain network affected in a variety of psychiatric and neurological conditions. In particular, several structures in the temporal lobe (e.g., the amygdala and the superior temporal sulcus) and in the frontal lobe (e.g., ventromedial frontal cortex) are thought to be either functionally or structurally affected in SCZ (Aleman and Kahn, 2005; Bediou et al., 2007b), in SIB (Lawrie et al., 1999), and at early stages of AD (Braak and Braak, 1991) and FTD (Neary et al., 1998). The basal ganglia, which are affected by $\mathrm{PD}$, are also critical for social message recognition (Curzon, 1977). In our study, the relative severity of the deficit in each disease seems to be related to the degree of functional or structural disturbance of these neural networks.

\section{Emotion recognition and frontotemporal neurodegeneration}

The relative decline in emotion recognition performance in aMCI, $\mathrm{AD}$, and FTD suggests a link between emotion recognition and frontotemporal neurodegeneration. First, investigating emotion recognition in aMCI and mild $\mathrm{AD}$ elucidates the impact of neurodegenerative processes on specific neural systems, especially the amygdala, which is critical for emotion perception (Adolphs et al., 1994). In $\mathrm{AD}$ at the aMCI stage, neurofibrillary tangles mainly affect the rhinal cortex and the hippocampus, but lateral temporal neocortical areas involved in emotion recognition, such as the STS, are relatively spared. This phenomenon may explain the preservation of this function at the aMCI stage and the impairment at the mild dementia stage of $\mathrm{AD}$. Indeed, at this stage, neurofibrillary tangles reach external temporal neocortical regions including the STS (Brak and Braak, 1991), and patients experience social disinvestment and lose their autonomy. Thus, the evolution of emotion recognition abilities, which is preserved in aMCI and altered in mild AD, seems to coincide with the progression of the neuropathology toward neocortical areas and social withdrawal. Therefore, assessing emotion recognition abilities may help in detecting this conversion at an even earlier stage.

Second, the study of AD and FTD patients elucidates the additional impact of frontal neurodegeneration on emotion recognition. Prefrontal cortex "mirror neurons," which discharge when an individual performs an action and when he or she observes someone else performing the same action, may play an important role in social cognition, especially for the recognition of facial expression (Gallese and Rizzolatti, 2004). FTD patients, who present with structural lesions within these networks, exhibit greater deficits in social cognition in general (Lough et al., 2006) and in face processing in particular (Keane et al., 2002), including the processing of other socially relevant, changeable facial features such as gaze direction (Bediou et al., 2009a). A greater deficit in the frontal variant compared to the temporal variant of FTD (Rosen et al., 2004) further supports the critical role of the prefrontal cortex in emotion recognition.

In our study, both the $\mathrm{AD}$ and the FTD groups displayed deficits in emotion recognition (relative to controls). We also reported that FTD patients were impaired relative to AD patients $(p=0.002)$, who in turn tend to perform significantly worse than aMCI subjects $(p=0.09)$, which provides indirect support for a relationship between frontotemporal neurodegeneration and emotion recognition deficits (Bediou et al., 2009a; see also Lavenu and Pasquier, 2005; Spoletini et al., 2008). Moreover, the fact that gender recognition was preserved in all groups suggests that, on the contrary, posterior cortical areas are not affected by any of these disorders.

\section{Emotion recognition and dopaminergic neurotransmission}

The finding that emotion recognition is impaired to the same extent in both SCZ and PD patients and is unaffected by anti- or 
pro-dopamine medication seems to indicate that emotion recognition deficits are independent from the dopaminergic system, which tends to challenge the hypothesis of a link between dopaminergic neurotransmission and emotion recognition (Salgado-Pineda et al., 2005). This finding is, however, consistent with an inverted U-Shape relationship between dopamine levels and emotion recognition ability (Delaveau et al., 2005). Moreover, this finding also tells us that the deficit is not specifically related to either the mesocorticolimbic or the nigrostriatal pathways, which are differentially affected by SCZ and PD.

Patients with SCZ present with functional and structural deficits affecting temporal structures including the amygdala and STS and prefrontal regions involved in social cognition (Lawrie and Abukmeil, 1998). These dysfunctions affecting the mesolimbic dopaminergic pathways result in a moderate impairment in the frontotemporal processing of facial expressions in these patients (Bediou et al., 2007b). The stability of facial expression recognition deficits over the course of schizophrenia, its independence with regard to treatment and the presence of a deficit in unaffected siblings suggest that impaired emotion recognition has trait characteristics and may represent an intermediate phenotype or an endophenotype of schizophrenia. The amygdala has been implicated in the pathophysiology of SCZ, including an increased risk for SCZ (Aleman and Kahn, 2005). Amygdala dysfunctions may contribute to social cognition impairments, which have been reported in both SCZ-rem patients and in SIB (Toomey et al., 1999) and may be correlated with mild social disinvestment. PD involves marked functional and structural abnormalities in subcortical motor structures especially the basal ganglia and interconnected structures, including the amygdala and the orbitofrontal cortex (Braak et al., 2003). Early dysfunction of this network may be particularly critical for social message recognition.

\section{IMPLICATIONS FOR THE PATHOPHYSIOLOGY, DIAGNOSIS, AND TREATMENT OF NEUROPSYCHIATRIC DISORDERS}

Although the present results are based on a reanalysis of selected studies from our group, in which we used the same methods to investigate emotion and gender recognition abilities in various clinical and high-risk populations in comparison to age and sex-matched control groups with limited sample sizes, our transnosologic approach has potential clinical implications. First, the finding that emotion recognition is differentially impaired among individuals with similar behavioral, clinical, and social disturbances (e.g., MDD vs. SIB) has potential implications for early differential diagnosis and intervention. Second, the finding that emotion recognition is differentially impaired at different stages of the same chronological continua (e.g., aMCI vs. $\mathrm{AD}$ ) may help in the early diagnosis and planning of early intervention.

Cognitive and social dysfunctions are common to a wide range of disorders such that the clear diagnosis often remains challenging. For instance, a patient presenting with depressive symptoms may cause the physician to suspect the first symptoms of schizophrenia. Although social withdrawal is common to both disorders, in our study, emotion recognition abilities were impaired in SCZ and their SIB but not in MDD (Bediou et al., 2007a).
Hence, assessing facial emotion recognition (FER) abilities may add valuable information in the differential diagnosis and initial treatment of these disorders. Another example concerns the similarities and therefore the difficulties in differentiating between SCZ that evolves toward dementia and FTD that begins with behavioral disorders compatible with SCZ (Kosmidis et al., 2008). FTD is also commonly misdiagnosed as bipolar disorder, given the overlap in symptoms. Again, adding facial expression recognition and gaze detection testing during the course of the differential diagnosis may be beneficial. No less challenging is the detection of the conversion from the aMCI to mild dementia stage of $\mathrm{AD}$, which may be subtle and subjective. A precise evaluation of a putative social dysfunction at that stage may be helpful in detecting social disinvestment and the progression toward a loss of autonomy (Bediou et al., 2009a).

The absence of significant emotion recognition deficits in MDD (and in aMCI, which is also known as prodromal AD) in our study is consistent with the preserved structural and functional integrity of the frontotemporal networks that are critical for emotion processing. However, this finding conflicts with numerous previous studies (Feinberg et al., 1986; Rubinow and Post, 1992; Persad and Polivy, 1993; Joormann and Gotlib, 2006; Joormann et al., 2007; Yoon et al., 2009a,b). Beyond variations in the methods, this discrepancy may reflect the heterogeneity of the MDD population and may be due more specifically to differences in patients' characteristics (e.g., symptom severity, illness duration, medication status), which are known to impact the ability to recognize emotions (Harmer et al., 2003, 2009; for a review of emotion recognition in MDD, see Bediou et al., 2009b). For instance, more severely depressed patients have displayed impairment in the more complex social emotion recognition tasks (Lee et al., 2005), whereas adolescents with bipolar disorders show impairments in the recognition of emotion in the faces of children but not adults (McClure et al., 2003). Moreover, MDD patients are often socially isolated, but this may be a purely behavioral consequence of the psychiatric disorder and not of a functional impairment of neural networks involved in emotion recognition.

\section{CONCLUSION}

In conclusion, emotion perception appears to be impaired in a variety of neurological and psychiatric disorders, although with different characteristics. In $\mathrm{AD}$, the impairment seems to coincide with the conversion toward dementia, whereas in SCZ, the perception of emotion is impaired at very early stages and even in unaffected siblings who are characterized by a genetic vulnerability to schizophrenia and have been found to share some cognitive impairments (albeit to a lesser extent) with their affected siblings. Hence, a deficit in the ability to recognize certain emotions may be a trait marker or an endophenotype of SCZ but a state marker for $\mathrm{AD}$. Taken together, these results have implications for researchers who are interested in the neurobiology of emotion perception mechanisms and can offer clinicians the possibility of building bridges between neurological and psychiatric disorders and to better understand the neurobiology (and time course) of common behavioral disorders. Given the small sample sizes used in the present study, further studies involving larger 
groups of subjects are needed before we can appreciate the clinical potential of such a transnosographic approach and add FER tasks to existing diagnostic test batteries. Although preliminary, this study also suggests that an approach based on overall emotion recognition may not be sufficiently sensitive to disentangle deficits in face-emotion processing among: (a) psychosis, (b) mood disorders, and (c) neurodegenerative diseases. Future meta-analyses

\section{REFERENCES}

Adolphs, R., Gosselin, F., Buchanan, T. W., Tranel, D., Schyns, P., and Damasio, A. R. (2005). A mechanism for impaired fear recognition after amygdala damage. Nature 433 , 68-72.

Adolphs, R., Tranel, D., Damasio, H., and Damasio, A. (1994). Impaired recognition of emotion in facial expressions following bilateral damage to the human amygdala. Nature 372, 669-672.

Aleman, A., and Kahn, R. S. (2005). Strange feelings: do amygdala abnormalities dysregulate the emotional brain in schizophrenia? Prog. Neurobiol. 77, 283-298.

American Psychiatric Association. (1994). Diagnostic and Statistical Manual of Mental Disorders, 4th Edn. Washington, DC: American Psychiatric Association.

Assogna, F., Pontieri, F. E., Caltagirone, C., and Spalletta, G. (2008). The recognition of facial emotion expressions in Parkinson's disease. Eur. Neuropsychopharmacol. 18, 835-848.

Bediou, B., Asri, F., Brunelin, J., KrolakSalmon, P., D'amato, T., Saoud, M., and Tazi, I. (2007a). Emotion recognition and genetic vulnerability to schizophrenia. Br. J. Psychiatry 191, 126-130.

Bediou, B., Henaff, M. A., Bertrand, O., Brunelin, J., D'amato, T., Saoud, M., and Krolak-Salmon, P. (2007b). Impaired fronto-temporal processing of emotion in schizophrenia. Neurophysiol. Clin. 37, 77-87.

Bediou, B., Franck, N., Saoud, M., Baudouin, J. Y., Tiberghien, G., Dalery, J., and D'amato, T. (2005a). Effects of emotion and identity on facial affect processing in schizophrenia. Psychiat. Res. 133, 149-157.

Bediou, B., Krolak-Salmon, P., Saoud, M., Henaff, M. A., Burt, M., Dalery, J., and D'amato, T. (2005b). Facial expression and sex recognition in schizophrenia and depression. Can. J. Psychiatry 50, 525-533.
Bediou, B., Ryff, I., Mercier, B., Milliery, M., Henaff, M. A., D'amato, T., Bonnefoy, M., Vighetto, A., and KrolakSalmon, P. (2009a). Impaired social cognition in mild Alzheimer disease. J. Geriatr. Psychiatry Neurol. 22, 130-140.

Bediou, B., Saoud, M., Harmer, C., and Krolak-Salmon, P. (2009b). Analyzing facial expressions in depression.

Bolte, S., and Poustka, F. (2003). The recognition of facial affect in autistic and schizophrenic subjects and their first-degree relatives. Psychol. Med. $33,907-915$.

Braak, H., and Braak, E. (1991). Neuropathological stageing of Alzheimer-related changes. Acta Neuropathol. 82, 239-259.

Braak, H., Del Tredici, K., Rub, U., De Vos, R. A., Jansen Steur, E. N., and Braak, E. (2003). Staging of brain pathology related to sporadic Parkinson's disease. Neurobiol. Aging 24, 197-211.

Bucks, R. S., and Radford, S. A. (2004). Emotion processing in Alzheimer's disease. Aging Ment. Health 8, 222-232.

Cadieux, N. L., and Greve, K. W. (1997). Emotion processing in Alzheimer's disease. J. Int. Neuropsychol. Soc. 3, 411-419.

Calder, A. J., Burton, A. M., Miller, P., and Young, A. J. (2001). A principal component analysis of facial expressions. Vision Res. 41, 1179-1208.

Calder, A. J., Keane, J., Manes, F., Antoun, N., and Young, A. W. (2000). Impaired recognition and experience of disgust following brain injury. Nat. Neurosci. 3, 1077-1078.

Canli, T., Cooney, R. E., Goldin, P., Shah, M., Sivers, H., Thomason, M. E., Whitfield-Gabrieli, S., Gabrieli, J. D., and Gotlib, I. H. (2005). Amygdala reactivity to emotional faces predicts improvement in major depression. Neuroreport 16, 1267-1270.

Chan, S. W., Norbury, R., Goodwin, G. M., and Harmer, C. J. (2009). Risk for depression and neural responses to fearful facial expressions of emotion. Br. J. Psychiatry 194, 139-145. Evol. Psychiatr. 74, 79-91.

should address how face-emotion processing deficits are unique to the brain alterations in each of these populations.

\section{ACKNOWLEDGMENTS}

This study was supported by a grant to Benoit Bediou from the Conseil Scientifique de la Recherche, Centre Hospitalier Le Vinatier.

Clark, U. S., Neargarder, S., and CroninGolomb, A. (2008). Specific impairments in the recognition of emotional facial expressions in Parkinson's disease. Neuropsychologia 46 , 2300-2309.

Cohen, J. (1969). Statistical Power Analysis for the Behavioral Sciences, 1st Edn. New York: Academic Press.

Curzon, G. (1977). The biochemistry of the basal ganglia and Parkinson's disease. Postgrad. Med. J. 53, 719-725.

Dalgleish, T. (2004). The emotional brain. Nat. Rev. Neurosci. 5, 583-589.

Davidson, R. J., Putnam, K. M., and Larson, C. L. (2000). Dysfunction in the neural circuitry of emotion regulation - a possible prelude to violence. Science 289, 591-594.

Delaveau, P., Salgado-Pineda, P., Wicker, B., Micallef-Roll, J., and Blin, O. (2005). Effect of levodopa on healthy volunteers' facial emotion perception: an FMRI study. Clin. Neuropharmacol. 28, 255-261.

Deruelle, C., and Fagot, J. (2005). Categorizing facial identities, emotions, and genders: attention to high- and low-spatial frequencies by children and adults. J. Exp. Child Psychol. 90, 172-184.

Deutch, A. Y. (1993). Prefrontal cortical dopamine systems and the elaboration of functional corticostriatal circuits: implications for schizophrenia and Parkinson's disease. J. Neural Transm. 91, 197-221.

Dujardin, K., Blairy, S., Defebvre, L., Duhem, S., Noel, Y., Hess, U., and Destee, A. (2004). Deficits in decoding emotional facial expressions in Parkinson's disease. Neuropsychologia 42, 239-250.

Eimer, M., and Holmes, A. (2007). Event-related brain potential correlates of emotional face processing. Neuropsychologia 45, 15-31.

Feinberg, T. E., Rifkin, A., Schaffer, C. and Walker, E. (1986). Facial discrimination and emotional recognition in schizophrenia and affective disorders. Arch. Gen. Psychiatry 43, 276-279.
Fu, C. H., Williams, S. C., Brammer, M. J., Suckling, J., Kim, J., Cleare, A. J., Walsh, N. D., Mitterschiffthaler, M. T., Andrew, C. M., Pich, E. M., and Bullmore, E. T. (2007). Neural responses to happy facial expressions in major depression following antidepressant treatment. Am. J. Psychiatry 164, 599-607.

Fu, C. H., Williams, S. C., Cleare, A. J., Brammer, M. J., Walsh, N. D., Kim, J., Andrew, C. M., Pich, E. M., Williams, P. M., Reed, L J., Mitterschiffthaler, M. T., Suckling, J., and Bullmore, E. T. (2004). Attenuation of the neural response to sad faces in major depression by antidepressant treatment: a prospective, event-related functional magnetic resonance imaging study. Arch. Gen. Psychiatry 61, 877-889.

Gallese, V. C. K., and Rizzolatti, G. (2004). A unifying view of the basis of social cognition. Trends Cogn. Sci. (Regul. Ed.) 8, 396-403.

Germine, L. T., and Hooker, C. I. (2011). Face emotion recognition is related to individual differences in psychosis-proneness. Psychol. Med. 41, 937-947.

Gibb, W. R., and Lees, A. J. (1989). The significance of the Lewy body in the diagnosis of idiopathic Parkinson's disease. Neuropathol. Appl. Neurobiol. 15, 27-44.

Gur, R. C., Erwin, R. J., Gur, R. E., Zwil, A. S., Heimberg, C., and Kraemer, H. C. (1992). Facial emotion discrimination: II. Behavioral findings in depression. Psychiatry Res. 42, 241-251.

Harmer, C. J., Goodwin, G. M., and Cowen, P. J. (2009). Why do antidepressants take so long to work? A cognitive neuropsychological model of antidepressant drug action. Br. J. Psychiatry 195, 102-108.

Harmer, C. J., Hill, S. A., Taylor, M. J. Cowen, P. J., and Goodwin, G. M. (2003). Toward a neuropsychological theory of antidepressant drug action: increase in positive emotional bias after potentiation of norepinephrine activity. Am. J. Psychiatry 160, 990-992. 
Haxby, J. V., Hoffman, E. A., and Gobbini, M. I. (2000). The distributed human neural system for face perception. Trends Cogn. Sci. (Regul. Ed.) 4, 223-233.

Hedges, L., and Olkin, I. (1985). Statistical Methods for Meta-Analysis. New York: Academic Press.

Heimberg, C., Gur, R. E., Erwin, R. J., Shtasel, D. L., and Gur, R. C. (1992). Facial emotion discrimination .3. Behavioral findings in schizophrenia. Psychiatry Res. 42, 253-265.

Heuer, K., Lange, W. G., Isaac, L., Rinck, M., and Becker, E. S. (2010). Morphed emotional faces: emotion detection and misinterpretation in social anxiety. $J$. Behav. Ther. Exp. Psychiatry 41, 418-425.

Ioannides, A. A., Poghosyan, V., Dammers, J., and Streit, M. (2004). Real-time neural activity and connectivity in healthy individuals and schizophrenia patients. Neuroimage 23, 473-482.

Isaac, L., and Lincoln, A. (2011). Featural versus configural face processing in a rare genetic disorder: Williams syndrome. J. Intellect. Disabil. Res. 55, 1034-1042.

Joormann, J., and Gotlib, I. H. (2006). Is this happiness $\mathrm{i}$ see? Biases in the identification of emotional facial expressions in depression and social phobia. J. Abnorm. Psychol. 115, 705-714.

Joormann, J., Talbot, L., and Gotlib, I. H. (2007). Biased processing of emotional information in girls at risk for depression. J. Abnorm. Psychol. 116, 135-143.

Kanwisher, N., and Moskovitch, M. (2000). The cognitive neuroscience of face processing: an introduction. Cogn. Neuropsychol. 17, $1-11$.

Keane, J., Calder, A. J., Hodges, J. R., and Young, A. W. (2002). Face and emotion processing in frontal variant frontotemporal dementia. Neuropsychologia 40, 655-665.

Kohler, C. G., Anselmo-Gallagher, G., Bilker, W., Karlawish, J., Gur, R. E., and Clark, C. M. (2005). Emotion-discrimination deficits in mild Alzheimer disease. Am. J. Geriatr. Psychiatry 13, 926-933.

Kohler, C. G., Turner, T. H., Bilker, W. B., Brensinger, C. M., Siegel, S. J., Kanes, S. J., Gur, R. E., and Gur, R. C. (2003). Facial emotion recognition in schizophrenia: intensity effects and error pattern. Am. J. Psychiatry 160, 1768-1774.
Kohler, C. G., Turner, T. H., Gur, R. E., and Gur, R. C. (2004). Recognition of facial emotions in neuropsychiatric disorders. CNS Spectr. 9, 267-274.

Kosmidis, M. H., Aretouli, E., Bozikas, V. P., Giannakou, M., and Ioannidis, P. (2008). Studying social cognition in patients with schizophrenia and patients with frontotemporal dementia: theory of mind and the perception of sarcasm. Behav. Neurol. 19, 65-69.

Krolak-Salmon, P., Fischer, C., Vighetto, A., and Mauguiere, F. (2001). Processing of facial emotional expression: spatio-temporal data as assessed by scalp event-related potentials. Eur. J. Neurosci. 13, 987-994.

Krolak-Salmon, P., Henaff, M. A., Isnard, J., Tallon-Baudry, C., Guenot, M., Vighetto, A., Bertrand, O., and Mauguiere, F. (2003). An attention modulated response to disgust in human ventral anterior insula. Ann. Neurol. 53, 446-453.

Krolak-Salmon, P., Hénaff, M. A., Vighetto, A., Bauchet, F., Bertrand, O., Mauguiere, F., and Isnard, J. (2006). Experiencing and detecting happiness in humans: the role of the supplementary motor area. Ann. Neurol. 59, 196-199.

Krolak-Salmon, P., Henaff, M. A., Vighetto, A., Bertrand, O., and Mauguiere, F. (2004). Early amygdala reaction to fear spreading in occipital, temporal, and frontal cortex: a depth electrode ERP study in human. Neuron 42, 665-676.

Lachenal-Chevallet, K., Bediou, B., Bouvard, M., Thobois, S., Broussolle, E., Vighetto, A., and Krolak-Salmon, P. (2006). Emotional facial expression recognition impairment in Parkinson disease. Psychol. Neuropsychiatr. Vieil. 4, 61-67.

Lavenu, I., and Pasquier, F. (2005). Perception of emotion on faces in frontotemporal dementia and Alzheimer's disease: a longitudinal study. Dement. Geriatr. Cogn. Disord. 19, 37-41.

Lavenu, I., Pasquier, F., Lebert, F., Petit, H., and Van Der Linden, M. (1999). Perception of emotion in frontotemporal dementia and Alzheimer disease. Alzheimer Dis. Assoc. Disord. 13, 96-101.

Lawrence, A. D., Goerendt, I. K., and Brooks, D. J. (2007). Impaired recognition of facial expressions of anger in Parkinson's disease patients acutely withdrawn from dopamine replacement therapy. Neuropsychologia 45, 65-74.
Lawrence, N. S., Williams, A. M., Surguladze, S., Giampietro, V., Brammer, M. J., Andrew, C., Frangou, S., Ecker, C., and Phillips, M. L. (2004). Subcortical and ventral prefrontal cortical neural responses to facial expressions distinguish patients with bipolar disorder and major depression. Biol. Psychiatry 55, 578-587.

Lawrie, S. M., and Abukmeil, S. S. (1998). Brain abnormality in schizophrenia. A systematic and quantitative review of volumetric magnetic resonance imaging studies. Br. J. Psychiatry 172, 110-120.

Lawrie, S. M., Whalley, H., Kestelman, J. N., Abukmeil, S. S., Byrne, M., Hodges, A., Rimmington, J. E., Best, J. J., Owens, D. G., and Johnstone, E. C. (1999). Magnetic resonance imaging of brain in people at high risk of developing schizophrenia. Lancet 353, 30-33.

Lee, L., Harkness, K. L., Sabbagh, M. A., and Jacobson, J. A. (2005). Mental state decoding abilities in clinical depression. J. Affect. Disord. 86, 247-258.

Lewis, S. F., and Garver, D. L. (1995) Treatment and diagnostic subtype in facial affect recognition in schizophrenia. J. Psychiatr. Res. 29, 5-11.

Lough, S., Kipps, C. M., Treise, C., Watson, P., Blair, J. R., and Hodges, J. R. (2006). Social reasoning, emotion and empathy in frontotemporal dementia. Neuropsychologia 44, 950-958.

Mandal, M. K., Pandey, R., and Prasad, A. B. (1998). Facial expressions of emotions and schizophrenia: a review. Schizophr. Bull. 24, 399-412.

McClure, E. B., Pope, K., Hoberman, A. J., Pine, D. S., and Leibenluft, E. (2003). Facial expression recognition in adolescents with mood and anxiety disorders. Am. J. Psychiatry 160, 1172-1174.

McKhann, G., Drachman, D., Folstein, M., Katzman, R., Price, D., and Stadlan, E. M. (1984). Clinical diagnosis of Alzheimer's disease: report of the NINCDS-ADRDA work group under the auspices of department of health and human services task force on Alzheimer's disease. Neurology 34, 939-944.

Mikhailova, E. S., Vladimirova, T. V., Iznak, A. F., Tsusulkovskaya, E. J., and Sushko, N. V. (1996). Abnormal recognition of facial expression of emotions in depressed patients with major depression disorder and schizotypal personality disorder. Biol. Psychiatry 40, 697-705.
Neary, D., Snowden, J. S., Gustafson, L., Passant, U., Stuss, D., Black, S., Freedman, M., Kertesz, A., Robert, P. H., Albert, M., Boone, K., Miller, B. L., Cummings, J., and Benson, D. F. (1998). Frontotemporal lobar degeneration: a consensus on clinical diagnostic criteria. Neurology 51, 1546-1554.

Persad, S. M., and Polivy, J. (1993). Differences between depressed and nondepressed individuals in the recognition of and response to facial emotional cues. J. Abnorm. Psychol. 102, 358-368.

Petersen, R. C., Smith, G. E., Waring, S. C., Ivnik, R. J., Tangalos, E. G., and Kokmen, E. (1999). Mild cognitive impairment: clinical characterization and outcome. Arch. Neurol. 56, 303-308.

Phillips, L. K., and Seidman, L. J. (2008). Emotion processing in persons at risk for schizophrenia. Schizophr. Bull. 34, 888-903.

Phillips, M. I., and David, A. S. (2003). Understanding the neurobiology of emotion perception: implications for psychiatry. Br. J. Psychiatry 182, 190-192.

Poreh, A. M., Whitman, R. D., Weber, M., and Ross, T. (1994). Facial recognition in hypothetically schizotypic college students. The role of generalized poor performance. J. Nerv. Ment. Dis. 182, 503-507.

Pourtois, G., and Vuilleumier, P. (2006). Chapter 4 Dynamics of emotional effects on spatial attention in the human visual cortex. Prog. Brain Res. 156, 67-91.

Pourtois, G., and Vuilleumier, P. (2010). Modulation of face processing by emotional expression during intracranial recordings in right fusiform cortex and amygdala. Int. J. Psychophysiol. 77, 234-234.

Rosen, H. J., Pace-Savitsky, K., Perry, R. J., Kramer, J. H., Miller, B. L., and Levenson, R. W. (2004). Recognition of emotion in the frontal and temporal variants of frontotemporal dementia. Dement. Geriatr. Cogn. Disord. 17, 277-281.

Roudier, M., Marcie, P., Grancher, A. S., Tzortzis, C., Starkstein, S., and Boller, F. (1998). Discrimination of facial identity and of emotions in Alzheimer's disease. J. Neurol. Sci. 154, 151-158.

Rubinow, D. R., and Post, R. M. (1992). Impaired recognition of affect in facial expression in depressed patients. Biol. Psychiatry 31, 947-953.

Salgado-Pineda, P., Delaveau, P., Blin, O., and Nieoullon, A. (2005). 
Dopaminergic contribution to the regulation of emotional perception. Clin. Neuropharmacol. 28, 228-237.

Seiferth, N. Y., Pauly, K., Habel, U., Kellermann, T., Shah, N. J., Ruhrmann, S., Klosterkotter, J., Schneider, F., and Kircher, T. (2008). Increased neural response related to neutral faces in individuals at risk for psychosis. Neuroimage 40, 289-297.

Shayegan, D. K., and Stahl, S. M. (2005). Emotion processing, the amygdala, and outcome in schizophrenia. Prog. Neuropsychopharmacol. Biol. Psychiatry.

Sheline, Y. I., Barch, D. M., Donnelly, J. M., Ollinger, J. M., Snyder, A. Z., and Mintun, M. A. (2001). Increased amygdala response to masked emotional faces in depressed subjects resolves with antidepressant treatment: an fMRI study. Biol. Psychiatry 50, 651-658.

Spoletini, I., Marra, C., Di Iulio, F., Gianni, W., Sancesario, G., Giubilei, F., Trequattrini, A., Bria, P., Caltagirone, C., and Spalletta, G. (2008). Facial emotion recognition deficit in amnestic mild cognitive impairment and Alzheimer disease. Am. J. Geriatr. Psychiatry 16, 389-398.

Sprengelmeyer, R., Young, A.W., Pundt, I., Sprengelmeyer, A., Calder, A. J.,
Berrios, G., Winkel, R., Vollmoeller, W., Kuhn, W., Sartory, G., and Przuntek, H. (1997). Disgust implicated in obsessive-compulsive disorder. Proc. R Soc. Lond. B Biol. Sci. 264, 1767-1773.

Streit, M., Ioannides, A., Sinnemann, T., Wolwer, W., Dammers, J., Zilles, K., and Gaebel, W. (2001). Disturbed facial affect recognition in patients with schizophrenia associated with hypoactivity in distributed brain regions: a magnetoencephalographic study. Am. J. Psychiatry 158, 1429-1436.

Streit, M., Wolwer, W., and Gaebel, W. (1997). Facial-affect recognition and visual scanning behaviour in the course of schizophrenia. Schizophr. Res. 24, 311-317.

Toomey, R., and Schuldberg, D. (1995). Recognition and judgment of facial stimuli in schizotypal subjects. J. Commun. Disord. 28, 193-203.

Toomey, R., Seidman, L. J., Lyons, M. J., Faraone, S. V., and Tsuang, M. T. (1999). Poor perception of nonverbal social-emotional cues in relatives of schizophrenic patients. Schizophr. Res. 40, 121-130.

Tost, H., Alam, T., and MeyerLindenberg, A. (2010). Dopamine and psychosis: theory, pathomechanisms and intermediate phenotypes. Neurosci. Biobehav. Rev. 34, 689-700.

Van Rijn, S., Aleman, A., Swaab, H., and Kahn, R. S. (2005). Neurobiology of emotion and high risk for schizophrenia: role of the amygdala and the X-chromosome. Neurosci. Biobehav. Rev. 29, 385-397.

Van 'T Wout, M., Aleman, A., Kessels, R. P., Laroi, F., and Kahn, R. S. (2004). Emotional processing in a non-clinical psychosis-prone sample. Schizophr. Res. 68, 271-281.

Vuilleumier, P., and Pourtois, G. (2007). Distributed and interactive brain mechanisms during emotion face perception: evidence from functional neuroimaging. Neuropsychologia 45, 174-194.

Wolwer, W., Streit, M., Polzer, U., and Gaebel, W. (1996). Facial affect recognition in the course of schizophrenia. Eur. Arch. Psychiatry Clin. Neurosci. 246, 165-170.

Yoon, K. L., Joormann, J., and Gotlib, I. H. (2009a). Judging the Intensity of facial expressions of emotion: depression-related biases in the processing of positive affect. J. Abnorm. Psychol. 118, 223-228.

Yoon, K. L., Joormann, J., and Gotlib, I. H. (2009b). Judging the intensity of facial expressions of emotion: depression-related biases in the processing of positive affect. J. Abnorm. Psychol. 118, 223-228.
Zuroff, D. C., and Colussy, S. A. (1986). Emotion recognition in schizophrenic and depressed inpatients. J. Clin. Psychol. 42, 411-417.

Conflict of Interest Statement: The authors declare that the research was conducted in the absence of any commercial or financial relationships that could be construed as a potential conflict of interest.

Received: 08 December 2011; accepted: 16 March 2012; published online: 03 April 2012.

Citation: Bediou B, Brunelin J, d'Amato $T$, Fecteau S, Saoud M, Hénaff $M-A$ and Krolak-Salmon P (2012) A comparison of facial emotion processing in neurological and psychiatric conditions. Front. Psychology 3:98. doi: 10.3389/fpsyg.2012.00098

This article was submitted to Frontiers in Emotion Science, a specialty of Frontiers in Psychology.

Copyright (C) 2012 Bediou, Brunelin, d'Amato, Fecteau, Saoud, Hénaff and Krolak-Salmon. This is an open-access article distributed under the terms of the Creative Commons Attribution Non Commercial License, which permits noncommercial use, distribution, and reproduction in other forums, provided the original authors and source are credited. 